\title{
Design of Multiplier for Medical Image Compression Using Urdhava Tiryakbhyam Sutra
}

\author{
Suma $^{1}$, V. Sridhar ${ }^{2}$ \\ ${ }^{1}$ Vidya Vikas Institute of Engineering \& Technology, Mysore, India \\ ${ }^{2}$ PES College of Enginnering, Mandya, India
}

\begin{tabular}{l} 
Article Info \\
\hline Article history: \\
Received Oct 12, 2015 \\
Revised Jan 5, 2016 \\
Accepted Jan 20, 2016 \\
\hline
\end{tabular}

Keyword:

Bit loading algorithm Power allocation algorithm Resource allocation Wireless network

\begin{abstract}
Compressing the medical images is one of the challenging areas in healthcare industry which calls for an effective design of the compression algorithms. The conventional compression algorithms used on medical images doesn't offer enhanced computational capabilities with respect to faster processing speed and is more dependent on hardware resources. The present paper has identified the potential usage of Vedic mathematics in the form of Urdhava Tiryakbhyam sutra, which can be used for designing an efficient multiplier that can be used for enhancing the capabilities of the existing processor to generate enhance compression experience. The design of the proposed system is discussed with respect to 5 significant algorithms and the outcome of the proposed study was testified with heterogeneous samples of medical image to find that proposed system offers approximately $57 \%$ of the reduction in size without any significant loss of data.
\end{abstract}

Copyright (C) 2016 Institute of Advanced Engineering and Science. All rights reserved.

\section{Corresponding Author:}

Suma,

Vidya Vikas Institute of Engineering and Technology,

Mysore, India

Email: sumaaldur@gmail.com

\section{INTRODUCTION}

With the advancement of the modernized technique for diagnosis of the diseases, the healthcare system has undergone a revolutionary change by adopting medical image processing. Although medical image processing is a part of digital image processing, but it could be said as an advance applications of digital image processing as the complexities and charecteristics associated with medical images are completely different from natural images [1]. Usually, medical images are captured using various existing image capturing devices e.g. X-ray, CT-scan, MRI etc. Such medical images posses valuable information which is used for mainly three purposes e.g. i) diagnosing the diseases of the subject, ii) storing the images in the database for further clinical studies for medical students, and iii) research and development of novel ideas to mitigate the diagnosed disease. Unfortunately, the medical images (e.g. CT scan images, MRI images etc.) gives information embedded in maximum resolution per slices of the data capture and thereby increasing the size of image from gigabytes to terabytes for just one medical report. However, with the data storage being cheaply available in forms of distributed servers and cloud environment, storage is never a problem for the medical images. The entire problem shoots up when the circumstances calls for transmitting the medical images. Such circumstance is when the healthcare industry uses telemedicine [2]. The concept of telemedicine allows the patient and physician to interact with each other using the network resources which always has limitations of bandwidth. Such applications of telemedicine works in different principle e.g. i) doctor would like to access the medical image using downlink transmission, or ii) doctor could like to perform some processing on medical images that resides on different machines along with other simultaneous data transfer. Hence, such applicability of telemedicine as well as upcoming robotic surgery calls for highly robust compression algorithm with better response rate. Here the term response will mean how fast the 
algorithm can perform encoding of the streams of medical data and generate reconstructed image without losing significant information of clinical value. From past decade, there are various algorithms and techniques introduced by various researchers for carrying out medical image compression, where majority of the studies have their own advantages and disadvantages. However, it is found that existing studies are much focused on size reduction and less focus on computational capabilities e.g. processor speed, high resolution, slicing the components of image etc. Hence, in this regards, we came across the evolution of Vedic mathematics and its potential application that can be readily applicable in signal processing [3],[4]. The principle of Vedic mathematics is based on the 16 principle that are originally termed as Sutra [5]. In order to understand the applicability of Vedic mathematics, we will need to understand that conventional compression techniques don't focus on enhancing the processor speed owing to lack of valuable concepts of multiplier. We found that Vedic mathematics offers a specific sutra for multiplier called as Urdhva Tiryakbhyam sutra, which can be highly useful for designing a new multiplier. This Vedic multiplier will be assisting is performing compression with faster speed as compared with the conventional schemes. Therefore, we make use of this multiplier in designing a processor friendly and cost effective medical compression algorithm. The prime purpose of the proposed system is to ensure reduction of computational time as well as retaining higher contents of the image contents. Section 2 discusses about the research methodology and research and discussion has been discusses in Section 3. And finally Section 4 makes some concluding remarks.

\subsection{Background}

In the recent technologies, the size of hard disks of computer and network systems has increased, but the use of medical image continues to grow exponentially at the same time require a better image and better visual quality of image. These motivate the need of compression methods. In these professional technologies, such as medical images, large amounts of data's are required each and every day. So image compression has becomes a necessary to ensure that their storage of data as well as transfer in insecure medium networks.

The work of Urbaniak et al. [6] basically focused on the improvement of the diagnostic important images for diagnostic purpose and to get better compression. Here the author investigated the image compression of artifacts resulting from JPEG 2000 and JPEG. The performance parameters selected for the study are PSNR, quality of image, Structural Similarity Quality Measure (SSIM), and compression ratio. This result indicates that the compression ratio based on ROC gives better visual quality as well as SSIM gives better performances. Saini et al. [7] have performed an experiment and gives a comparison in image compression like HAAR wavelet, Bi-orthogonal Wavelets, Daubechies Wavelets and Coiflets wavelets. These algorithms are performed and testing has done for different medical images to reduce the image size and less storage requirements and it is relevant to diagnostically important regions. The outcome of the study was found with better image quality with an effective compression ratio.

Nassiri et al. [8], done an experiment on medical image compression for diagnostically important regions using Discrete Wavelet Transforms. To minimize the total degradation and get a better compression ratio of images, the DWT compression includes hard and soft thresholding decomposition methods. The study was tested with grayscale thoracic cage image of size $512 \times 512$. The study of a volumetric diagnostically important region of medical images using 3-D listless embed block algorithm has been presented by the author Sudha et al. [9]. Here the author modified this algorithm using Set Partitioned Embedded Block Coder (SPECK) methods to get high inter band correlation. Here the MRI images are used for testing the purposes. This algorithm improves the compression ratio and as well as the improve the visual perception of images quality. John et al. [10] have presented a high security, high transmission system for transmitting medically, diagnostically important reports for military and very high secure environment data's using hardware implementations methods. The authors have carried out encryption of an image on FPGA [Virtex 5 XC5VLX110T] and have also implemented a 16-stage pipeline module for achieving an encryptions rate of $35.5 \mathrm{Gbit} / \mathrm{s}$ with 2140 configurable logic blocks. Tiwari et al. [11] have presented multiple approaches solve a problems of storing or transmitting large number of medical data or images using different algorithms like DCT, DWT and Compressive Sensing techniques. In this paper the author performed a compressing and reconstruction technique for MRI image, CT image and Ultrasound image and the author given comparisons of all the three different medical images by using different algorithms. Here, the performance parameters taken are PSNR, MSE, compression ratio, quality of image along with storage capacity. The work by Gupta et al. [12] have presents a method of lossless image compression for medical images using predictive coding techniques as well as integer wavelet transform based on minimum entropy techniques. This paper presents a hybrid image compression a technique that combines an integer wavelet and predictive algorithms to enhance the performance of lossless compression. The evaluation of this technique was done over greyscale medical image essentially using transformation technique along with 
predictive-based approach. The proposed medical image compression techniques offer a higher level of compression ratio and minimum entropy when it is applied to many several levels of test images.

The paper presented by Kunchigi et al. [13] have presented a study of a Vedic mathematics approach applying for the design of 2-D DCT applications in medical image processing. Here, the author uses an Urdhva Tiryagbhyam Vedic sutra for the multiplication techniques in DCT. This paper also studied different Vedic sutras used for multiplication techniques like, Nikhilam sutra, Urdhva-Tiryagbhya sutra. The results obtained in 3 different cases, like single digit Vedic sutra, two digit Vedic sutra and finally three digit Vedic sutra. At finally, the three digit Vedic sutra gives best and better result among the three. i.e., higher image quality as well as better image compression ratio. Sarala et al. [14] have presented a paper on image compression using multi-level 2-D DWT as well as Vedic mathematic methods. A traditional 2-D compression algorithm consumes more power as well as more memory for image compression. But by using the Vedic mathematics algorithm used for image compression and reconstruction gives a better image compression ratio as well as better visual quality of image. This method is very useful for medical images. Vedic multiplier uses half adder and full adder. This method of 4-level 2D DWT techniques attempts to increase the image resolution. The simulations are done using Matlab 2008a version as well as ModelSim 6.3 version and it is implemented using Xilinx and FPGA Spartan 3 kit. Least but not last, another approach by Sowjanya et al. [15], implemented an approach of 2-D DCT architecture using Reversible Vedic Adder approach. It is used to reduce the size of the image as well as in both 1-D and 2-D image processing applications. It can also be used to calculate the 1-D and 2-D DCT using minimum number of hardware components. Here, the author designed a novel method by using Xilinx ISE 12.3i, Verilog HDL tools. It consumes less power consumption as well as less memory due to replacement of adder with reversible Vedic mathematics approaches. This novel algorithm improves the overall system performance in image processing. Hence, although there are various categories of compression algorithms witnessed most recently in medical images, but very few of them are found to address the computational complexities. Therefore, there is a need of a study that could potentially address such computational issues.

\subsection{The Problem}

Usually, the size of the medical images is too large to store. Owing to the advancement in the medical studies, there are increasing dependencies associated with an efficient storage and cost effective transmission of the medical images. A medical image from Sky Scan x-ray device [16] generates images of size $8000 \times 8000$ pixels, which in nutshell leads to generation of $64 \mathrm{MB}$ of image data just for one slice of the CT scan image. Therefore, other sophisticated medical imaging devices like MRI can generate around 1.19 GB of image data, which are not only difficult to store but highly challenging to perform transmission. Hence, such problem calls for performing compression technique. At present, various computing tools e.g. CUDA [17], Qt-Threaded [18], OpenCL [19] etc are in use in the processing such massive size of the medical images. To some extent, the discussion of the problem associated with the medical image compression was done in our prior work [20]. The problems that have been identified for the proposed system are as follows:

- Less Focus on Computational Capability: The existing technique mainly focuses on performing compression by reducing the sizes of medical images of uniform dimensions. We strongly feel that focus on medical image compression should be also extended towards ensuring the computational capability of the system from the hardware viewpoint. Hence, there is a need of the system that can provide an efficient and hardware friendly standards to perform compression of medical images.

- Less Emphasis on Multipliers: Multipliers plays a critical role in digital image processing especially while performing compression. Efficient design of multiplier while performing compression always increases the speed of processor, which is extremely important in telemedicine. However, there is less work focused using conventional technique for adoption of multipliers in medical image compression. Even the usages of multipliers by using Vedic mathematics were only tested on VLSI or FPGA platforms with narrowed experimental studies. Hence, there is a need of an effective design of multiplier using cost effective computational approach to ensure processor performance while handling compression of sophisticated medical images for carrying out compression.

- Less Emphasis on data redundancies: It is found that majority of the existing techniques on medical image compression ignores considering elimination of redundant data. Although majority of the existing techniques uses lossless approach for performing compression, but it seems to ignore the data redundancies that results in either less PSNR values or non-supportability in colored image compression.

The above problems are the prime identification factors of the problems found after reviewing the existing system. Hence, the problem statement of the proposed study can be stated as - "It is a computationally challenging task to design a technique that ensures processor friendly as well as cost effective compression scheme on all sorts of medical images.". 


\subsection{The Proposed Solution}

The prime goal of the proposed system is to enhance the performance of medical image compression by applying an effective multiplier design motivated from Vedic mathematics. The design of the multiplier is done considering the algorithm called as Urdhava Tiryakbhyam sutra that is responsible for performing vertical and crosswise multiplication.

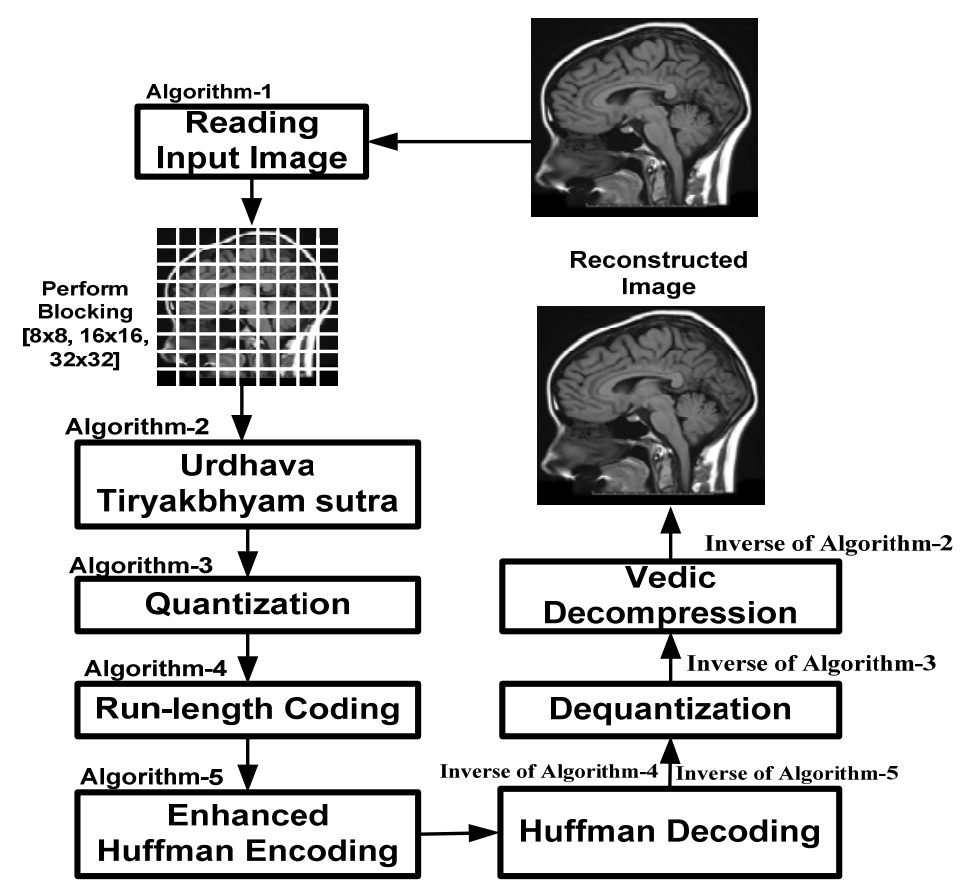

Figure 1. Schema of Proposed Medical Image Compression

The design of the proposed algorithm is based on a fact that addition of image elements by concurrent techniques will lead to partial products and this capability can be further strengthened by incorporating Urdhava Tiryakbhyam sutra to promote parallelism. The system allows simultaneous computation of summations of the elements being generated by the partial multiplication. Hence, such forms of multiplier do not depend on processor and its frequency of clock. In other language, the proposed approach of Vedic mathematics allows the system to execute the Vedic multiplier without any significant dependency on frequency of clock. Hence, the contribution of the proposed system can be briefed as,

- To read the medical image and is applicable on both grayscale as well as colored images.

- To apply multiple block-wise operation for the given image. We test it using block size of $8 \times 8,16 \times 16$, and $32 \times 32$.

- To apply the Urdhava Tiryakbhyam sutra on each test block for performing compression of the medical images.

- To address the redundancy problems while performing large medical image compression by using runlength coding.

- To generate the encoded image and check for its quality in the respective reconstructed image using PSNR and MSE.

- To check the consistency of the proposed technique on multiple type of medical images on standard datasets.

\section{RESEARCH METHOD}

The implementation of the proposed system is carried out using Matlab using normal 32 bit machine. The proposed system considers a medical image as an input and performs compression based on Urdhava Tiryakbhyam approach, which performs vertical and crossover multiplication in Vedic mathematics. In order to closely observe the processing time of the compression algorithms being applied, we choose to carry out this experiment on numerous machines with multiple processor of core-i5, dual core, AMD etc. In order to 
ease off the computational complexities associated with the processing of larger size of medical images, we consider converting the input image $\left(\mathrm{I}_{\text {orig }}\right)$ to grayscale. Our Algorithm-1 will show the basic step for performing this conversion that result in grayscale image ( $\left.\mathrm{I}_{\text {gray }}\right)$.

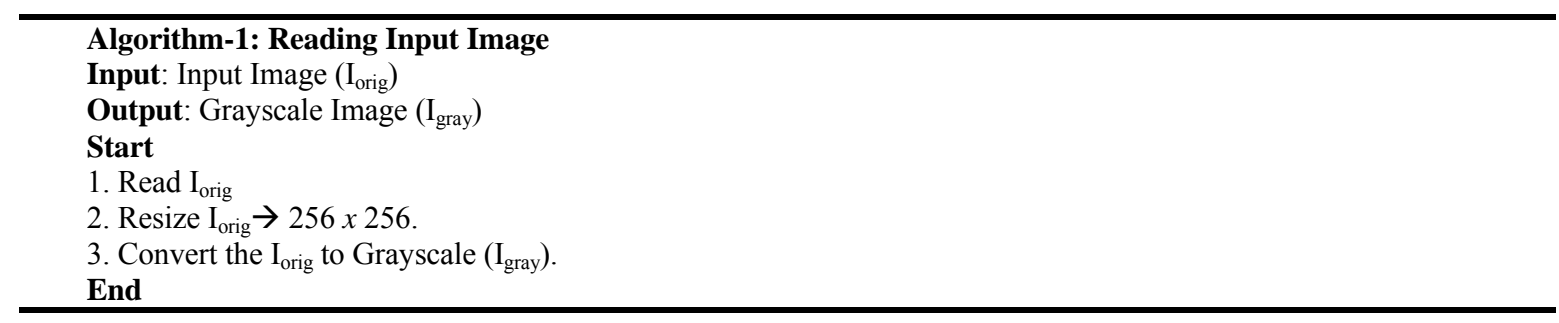

After obtaining the grayscale image $\left(\mathrm{I}_{\text {gray }}\right)$, the next step is to perform the compression technique using Vedic compression algorithm that is highlighted in Algorithm-2. The algorithm takes the input of $\mathrm{I}_{\text {gray }}$ and then performs the distinct block processing of either of the size $8 \times 8,16 \times 16$, and $32 \times 32$. The sizes of the blocks are fed to the system using the user interface and hence are of string type. According to the Algorithm2 , the size of the considered block is increased to double precision and stored in matrix R. The system will also increase the precision of $I_{\text {gray }}$ to double for better evaluation. The next phase of implementation will be to execute the operation of Vedic compression by applying Vedic multiplier. For this purpose, the size of the $\mathrm{I}_{\text {gray }}$ is evaluated and is mapped in a separate matrix of row and column elements. The algorithm also considers certain extra zero elements to the $\mathrm{I}_{\text {gray }}$ matrix and then it performs compression. In order to carry out a compression, the system designs a new function for Vedic multiplier (as shown in Line-5 of Algorithm-2), where $N$ is size of $X, T$ is transposition matrix, $p$ is a matrix with element $1-(N-1)$ and $q$ is a matrix with element 0 to $(N-1)$. Finally, the algorithm applies the Vedic Multiplier on the squared blocks of the image for a size R. The outcome of this algorithm is a compressed version of an image using Vedic Multiplier.

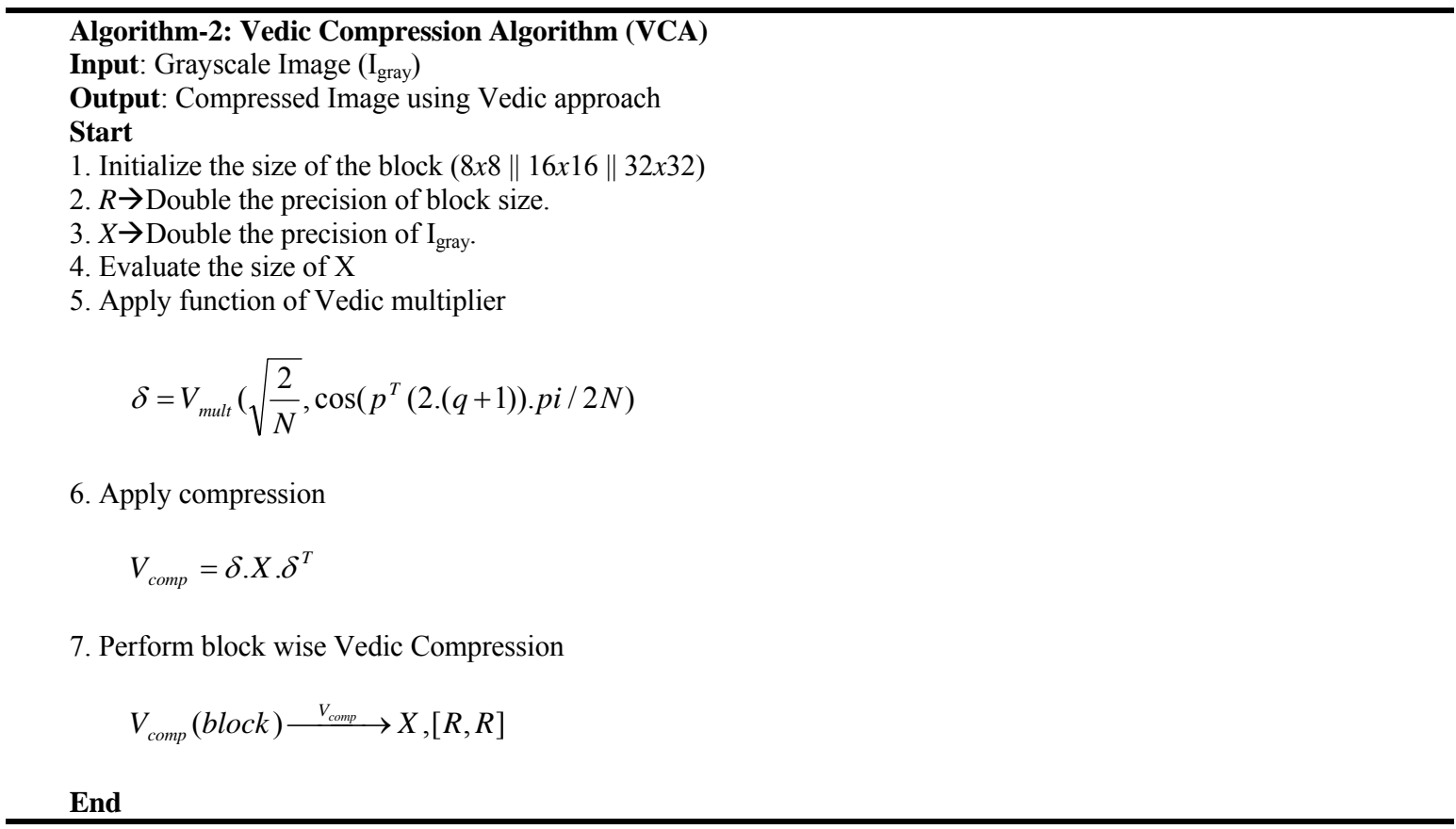

The outcome of the Algorithm-2 is subjected to the quantization technique. However, we prefer to perform the quantization in bit discrete stage compared to common style of applying quantization in image processing. Algorithm-3 highlights the steps being used for carrying out quantization over the compressed image (from Algorithm-2). We consider a threshold value $\mathrm{T}_{\mathrm{H}}=5$ for analysis purpose. The initial step of this algorithm mainly evaluates the size of the compressed image i.e. $\mathrm{V}_{\text {comp }}$ (block) and then it maps the size of it to 
a matrix with $\mathrm{P}$ (row) and $\mathrm{Q}$ (column). For all the elements of this matrix considering the maximum limits of $\mathrm{P}$ and $\mathrm{Q}$ elements, the proposed system attempts to compare the absolute value of compressed image i.e. $\mathrm{V}_{\text {comp }}$ (block) with the threshold $\mathrm{T}_{\mathrm{H}}$. Under any circumstances, if the absolute value of compressed image i.e. $\mathrm{V}_{\text {comp }}$ (block) is found to be within the limits of threshold $\mathrm{T}_{\mathrm{H}}$, than the system assign zero value to the compressed image and increases its count $k$ to read other block elements. The system also initialize the quantization value to be 8 and evaluates minimum and maximum arguments of compressed for making it applicable for equation shown in Line-15 of Algorithm-3. The outcome of this algorithm is a quantized image.

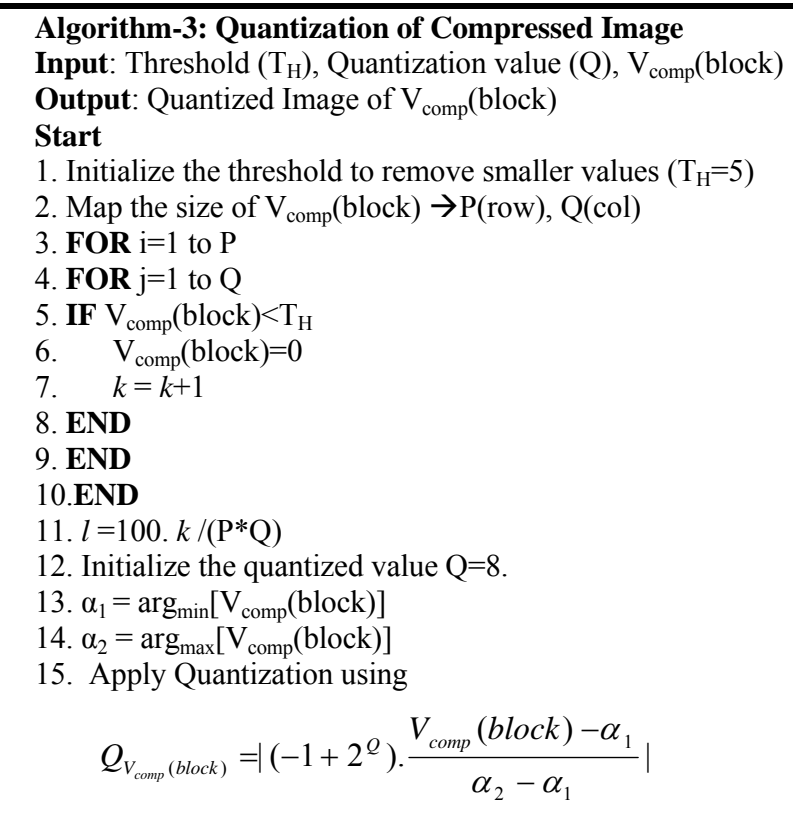

End

The outcome of Algorithm-3 i.e. quantized image is now subjected to Huffman Coding. The reason behind choosing dictionary based encoding mechanism e.g. Huffman coding is that it can perform compression of all categories of data. The Huffman coding can be termed as an entropy-based approach that is highly dependent on evaluation of the frequencies of array symbols. The potential of this coding scheme is precise identification of colors in medical images without any significant loss of valuable information. Moreover, at present, the medical image processing has already adopted such dictionary based encoding scheme in its lossless JPEG compression which is frequently adopted in medical imaging in the form of DICOM standards. However, we will choose to implement the Huffman Coding in a pattern different from conventional practices as shown in Algorithm-5. In this case, we choose to initialize the quantization value $\mathrm{Q}=8$ and performs zigzag scanning of the quantized image based on the concept of Urdhava Tiryakbhyam in Vedic mathematics. The algorithm chooses to perform the zigzag scanning for all the cases of squared block sizes $R$ of $8 \times 8$ or $16 \times 16$ or $32 \times 32$. Finally, the scanned reports of the blocks are mapped in a matrix (NM) with a specific row $\left(\mathrm{M}_{1}\right)$ and column size $\left(\mathrm{M}_{2}\right)$. A new matrix $(\mathrm{XZv})$ is formulated with reshaped structure of transposition of zigzag scanned image elements and NM. The next part of the implementation of Algorithm-5 will be to perform run length encoding mechanism that takes the input of XZv. The mechanism to perform Run Length Coding on new matrix (XZv) is shown in Algorithm-4 that generates a matrix Xrle to store the encoded values. However, it should be noted that Algorithm-4 is embedded with Algorithm-5 in Line-8.

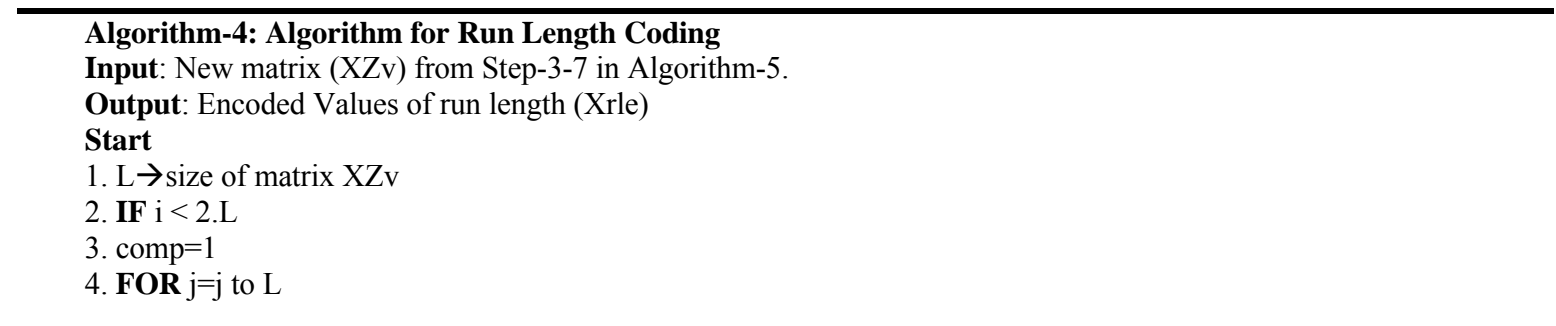




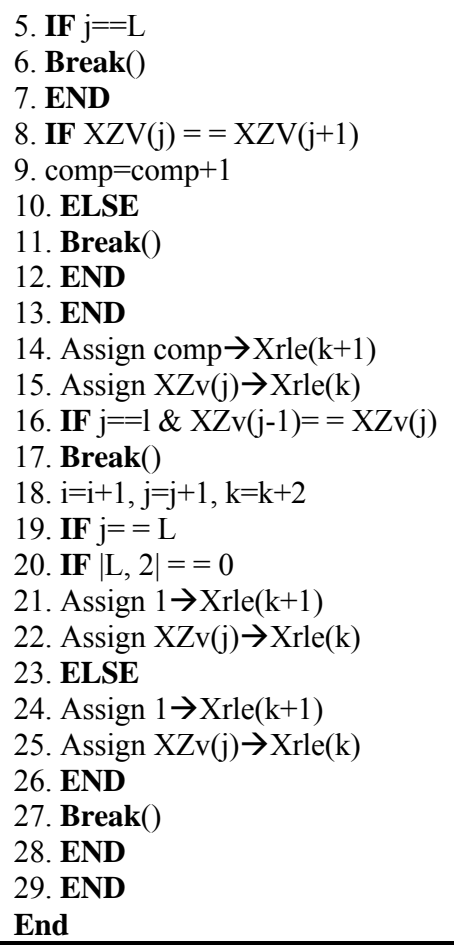

Hence, the execution of Algorithm-4 results in generation of a matrix (Xrle). The matrix comp creates a matrix of element starting from first element and with increment of 2 steps till the value that corresponds with the size of Xrle. Finally, we calculate probability of generated matrix for run length coding to reduce computational complexities of space factor and formulate a Huffman dictionary ranging from the value 0 to maximum probability factor. The design of the Algorithm-5 is as shown below.

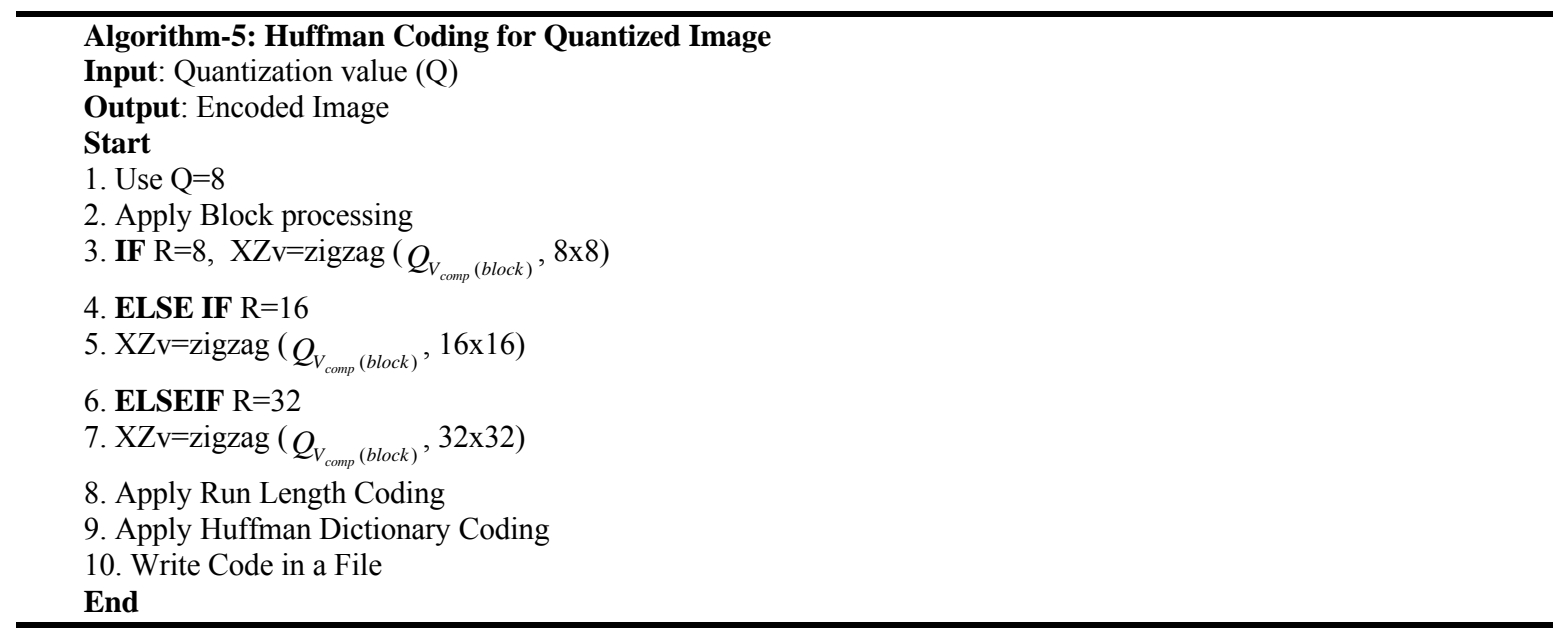

The implementation of the Algorithm-5 will generate code whose length (L) is evaluated and matrix repositing the code is round off to $\mathrm{L} / 8$ and is assigned to new matrix (Lcd). If the modulus of (L/8) is found to be less than the size 4 , the system recursively checks again of equivalence of the same with zero value. A positive case in this condition will increase the count of the round off values. Finally, the generated code is subjected to write in a new file for the purpose of decoding operation. The outcome of Algorithm-5 is compressed image, which will be saved in directory for further evaluation of reconstructed image. The steps of deconstruction follows the inverse process of the all the algorithms, where the outcomes were analyzed using original size of image (in bits), compressed size of image (in bits), compression ratio, Mean Squared Error, and PSNR. 


\section{RESULTS AND DISCUSSION}

The outcome of the proposed system has been tested with Cornell University dataset [21], where the performance parameters are mainly i) Size of Compressed Image, ii) Compression Ratio, iii) Mean Squared Error, and iv) Peak Signal-to-Noise ratio. The dataset consists of around 1000 MRI images, where we choose to evaluate both grayscale as colored medical images. However, this section of the manuscript will highlight only the significant cases of the sample dataset images and their outcomes when they were subjected to the discussed algorithms of this paper.

Table 1 highlights the sample images of the datasets that has been considered to perform evaluation of the proposed system. A closer look into the sample will show that proposed system has been analyzed considering multiple types of images with respect to color, sizes, and visual perceptibility factors. Table 2 shows the steps being undertaken for the first sample of medical images from the dataset as exhibited in Table 1. The visual outcome shows every step of compression and decompression involved in generating the reconstructed images. The Table 2 also highlights the type of the algorithm being implemented on each step of compression and decompression using Vedic mathematics. While in the process of generation of the reconstructed image, the system spontaneously checks original size of an image, size of compressed image, and compression ratio. The system then checks Mean Squared Error and Peak Signal-to-Noise ratio. The visual outcome shows that for every block size in increasing order, the visuality of the reconstructed image is well balanced. There is no blurriness or any fading effect even if the block sizes were increased for the purpose of compression. Therefore, the proposed system can offer better reconstruction of the compressed image without much loss of significant information which is of highest importance in pathological investigation.

Table 1. Samples considered for the testing

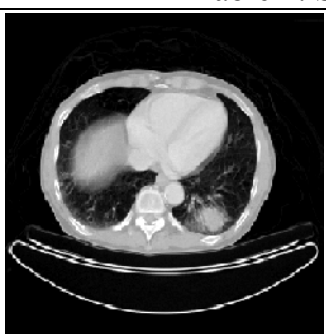

Size: $192 \mathrm{~KB}$

Dimension: 256 x 256

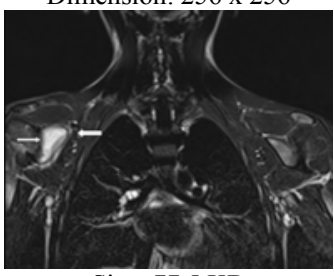

Size: $77.5 \mathrm{~KB}$

Dimension: 1280 x 996

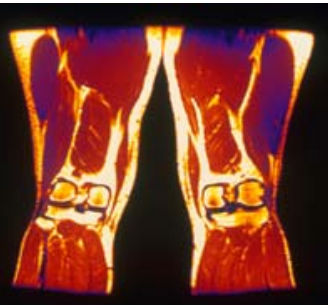

Size: $91.4 \mathrm{~KB}$

Dimension: $375 \times 375$

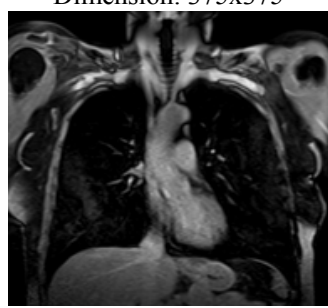

Size: $92.6 \mathrm{~KB}$ Dimension:500x468

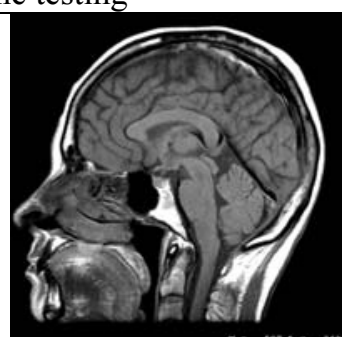

Size: $206 \mathrm{~KB}$

Dimension: 1024 x 1024

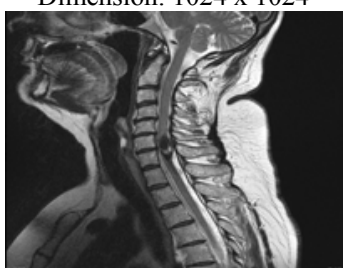

Size: $267 \mathrm{~KB}$

Dimension: 1365 x 1365

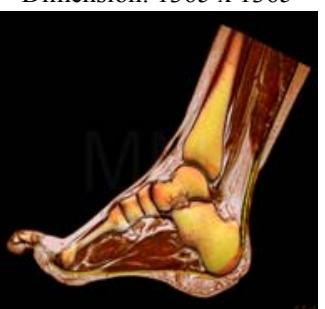

Size: $146 \mathrm{~KB}$

Dimension: 600x447

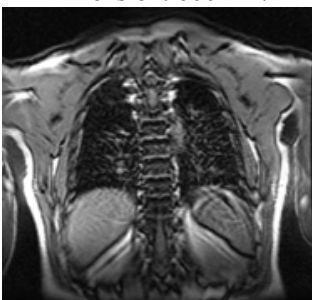

Size: $69.6 \mathrm{~KB}$

Dimension: 502x474 
Table 2 Visual Outcomes of Steps included in Generation of Reconstructed Image

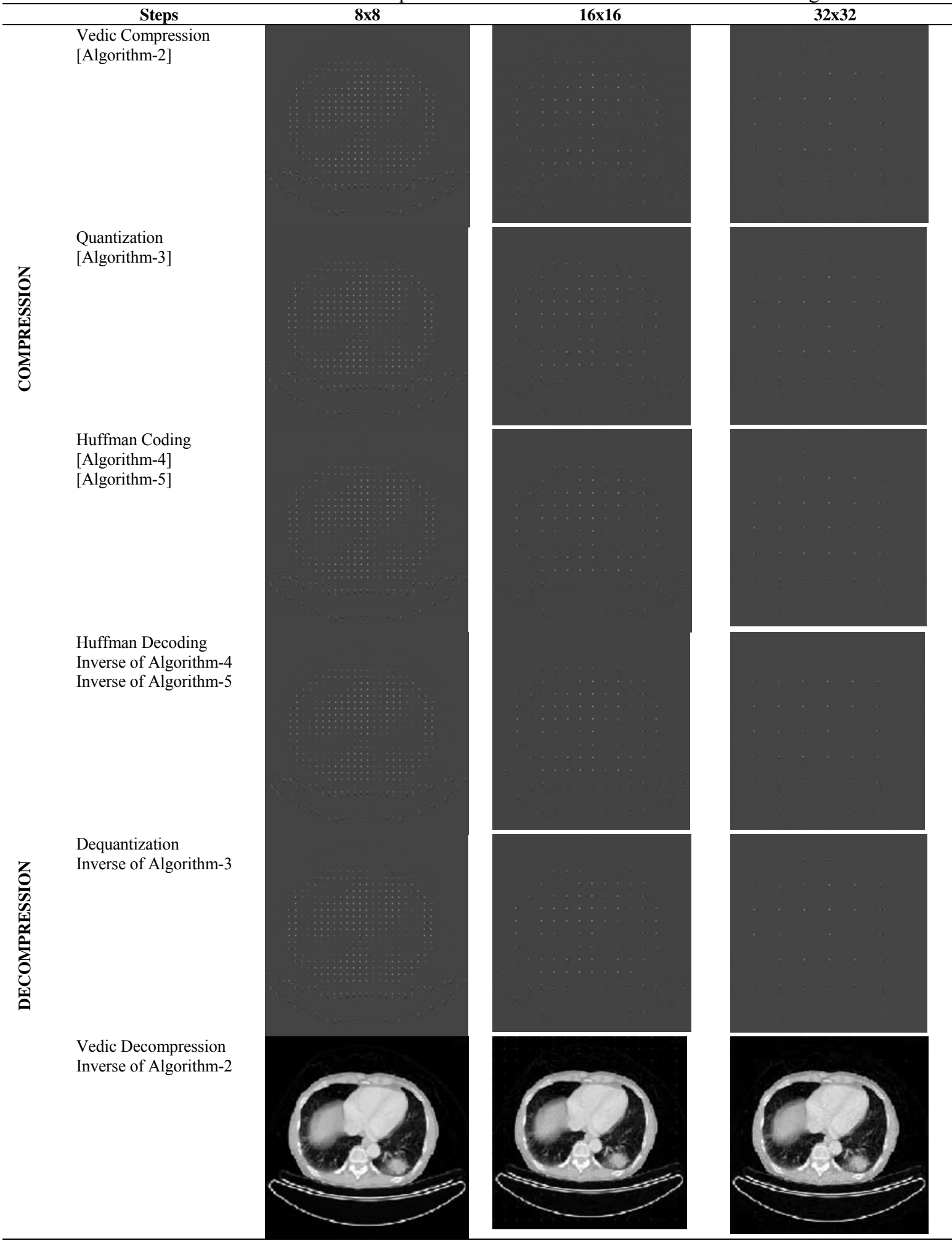

Table 3. Numerical Outcomes

\begin{tabular}{cccc}
\hline & \multicolumn{3}{c}{ Block Size } \\
Factors & $8 \times 8$ & $16 \times 16$ & $32 \times 32$ \\
\hline CS & 159608 & 151197 & 100444 \\
CR & 3.2848 & 3.4676 & 5.2197 \\
MSE & 2.3839 & 19.9899 & 18.2825 \\
PSNR & 44.358 & 35.1227 & 35.5105 \\
\hline
\end{tabular}

IJECE Vol. 6, No. 3, June 2016: 1140-1151 
Table 3 shows the numerical outcome of the proposed system considering the first test image from the samples shown in Table 1. The value shows that size of compressed image (CS) keeps on reducing with the increase of blocking size. Moreover, there is a smooth increment pattern in the Compression Ratio (CR) as well as Mean Squared Error (MSE) with the increase in block sizes, while PSNR is found to decline when the $16 \times 16$ blocks as compared to $8 \times 8$ block size. The outcome shows variable pattern of PSNR on different block size.

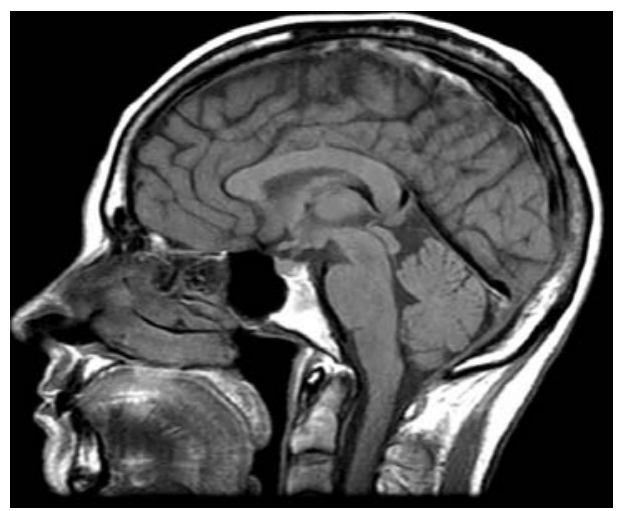

Figure 2. Second Image of Sample Dataset from Table 1

Table 4. Numerical Outcomes for Second Image in dataset

\begin{tabular}{cccc}
\hline & \multicolumn{3}{c}{ Block Size } \\
Factors & $8 \times 8$ & $16 \times 16$ & $32 \times 32$ \\
\hline CS & 186886 & 162472 & 160919 \\
CR & 2.8054 & 3.2269 & 3.2581 \\
MSE & 5.0103 & 12.2417 & 27.1282 \\
PSNR & 41.1321 & 37.2524 & 33.7966 \\
\hline
\end{tabular}

The first sample image holds a size of $192 \mathrm{~KB}$ and dimension of $256 \times 256$, hence, we have tested with the High Definition (HD) image of size 1024x1024 of size $206 \mathrm{~KB}$, which is shown in Figure 2. The numerical outcome for this HD image shows that size of compressed image drops with the increase of blocking size, while compression ratio as well as mean squared error is found to significantly increase its dimension with the increase of block size. The PSNR values for $8 \times 8$ block is found to be $41 \mathrm{db}$, which drops to $37 \mathrm{db}$ and $33 \mathrm{db}$ for the block size of $16 \times 16$ and $32 \times 32$. However, retaining the PSNR values within this range is quite acceptable with no loss of significant information during the compression operation executed by the algorithms of the proposed system. Our next set of evaluation was to check the similar consistency for colored images.

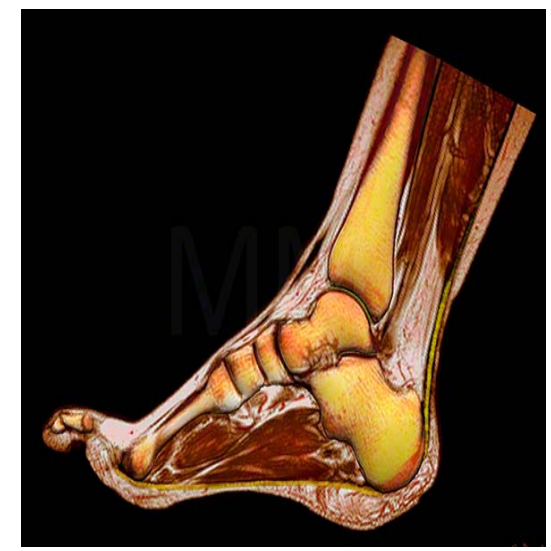

Figure 3. Sixth Image of Sample Dataset from Table 1 
Table 5. Numerical Outcomes for Sixth Image in dataset

\begin{tabular}{cccc}
\hline & & Block Size & \\
Factors & $8 \times 8$ & $16 \times 16$ & $32 \times 32$ \\
\hline CS & 124749 & 11146 & 120713 \\
CR & 4.2027 & 4.7044 & 4.3433 \\
MSE & 699.6571 & 725.1431 & 723.7361 \\
PSNR & 19.682 & 19.5266 & 19.535 \\
\hline
\end{tabular}

For evaluation of the compression techniques applied over colored image, we choose to showcase the numerical outcomes for the sixth sample image of the dataset shown in Table 1. The outcomes shows drop of compression ratio with the increase of block sizes, while a different pattern for compression ratio is being noticed. The compression ratio was found to increase from block size $8 \times 8$ to $16 \times 16$, but it (CR) experiences a minor drop in block size 32x32. Mean squared error has been seen with increasing trend with increase in block size, while PSNR is found to be quite uniform in all the versions of block sizes. The outcome evidently shows that proposed system offers similar performance gain in compressing the colored medical images too.

Table 6. Processing Speed \& \% of Compression Analysis

\begin{tabular}{lcc}
\hline & Speed (sec) & $\%$ of Compression (Approx) \\
\hline Image-1 & 2.5 & $47 \%$ \\
Image-2 & 2.9 & $21 \%$ \\
Image-3 & 3.5 & $32 \%$ \\
Image-4 & 1.5 & $46 \%$ \\
Image-5 & 2.5 & $22 \%$ \\
Image-6 & 3.6 & $18 \%$ \\
Image-7 & 3.2 & $21 \%$ \\
Image-8 & 1.2 & $41 \%$ \\
\hline
\end{tabular}

Table 6 shows the outcomes of the 8 sample images which is found with average of $31 \%$ of compression performance with 2.6 seconds of processing time recorded in core i5 processor. Our cumulative samples of 1000 images were found with average of 57\% of compression performance, which are highly optimal outcomes of the proposed system.

\section{CONCLUSION}

The complexities associated with the medical image were constantly being studied by various researchers in the past. This paper has reviewed some of the existing system towards performing compression of the medical images and found that there is a well scope to enhance the performance of compression. In the mean time, we came across various significant potential charecteristics of Vedic Mathematics, which provided powerful mathematical problem solving factors. Owing to few adoption in past for Vedic mathematics towards medical image compression, this paper contributes to address the research gap by introducing Vedic mathematics to enhance the performance of compression for medical images. The potential positive point of the proposed system is minimizing the core dependencies towards sophisticated processors for making them work in maximized frequency of clocks. This is one of the most significant outcomes of the proposed system as minimization of frequency of clock eventually results in higher consumption of power required for compressing the medical image. The paper has presented 5 algorithms that map with the stages of the compression methodology being adopted in the present study. The extensive outcome of the study shows that our proposed system can enhance 57\% of the compression performance with better retention of the PSNR.

\section{REFERENCES}

[1] R. S. Choras, "Image Processing and Communications Challenges 5," Springer Science \& Business Media, Computers, 2013.

[2] V. K Bairagi and A. M Sapkal, "ROI-based DICOM image compression for telemedicine," Sadhana-Indian Academy of Science, vol. 38, pp.123-131, 2013.

[3] K. M. Gaikwad and M. S. Chavan, "Vedic Mathematics for Digital Signal Processing Operations: A Review," International Journal of Computer Applications, vol/issue: 113(18), 2015.

[4] R. P. M. Sundari, et al., "Enhancing Multiplier Speed In Fast Fourier Transform Based On Vedic Mathematics," International Journal of VLSI design \& Communication Systems, vol/issue: 4(3), 2013.

[5] L. G. Moses and M. Thilagar, "VLSI Implementation of High Speed DSP algorithms using Vedic Mathematics," International Journal of Computer Communication and Information System, vol/issue: 2(1), 2010. 
[6] I. K. Urbaniak, et al., "The quest for "diagnostically lossless" medical image compression: A comparative study of objective quality metrics for compressed medical images," SPIE Medical Imaging: Image Perception, Observer Performance and Technology Assessment, vol. 9037, 2014.

[7] S. Saini and A. Mehta, "Experimental Comparison of Medical Image Compression Techniques," IEEE International Journal of Advanced Research in Computer Science and Software Engineering, vol/issue: 4(10), pp. 512-515, 2014.

[8] B. Nassiri, et al., "Study of Wavelet Based Medical Image Compression Techniques," International Journal of Engineering Science and Innovative Technology, vol/issue: 3(3), pp. 18-25, 2014.

[9] V. K. Sudha and R Sudhakar, "3-D Listless Embedded Block Coding Algorithm for Compression of Volumetric Medical Images," Journal of Scientific and Industrial Research, vol. 72, pp. 735-738, 2013.

[10] J. E. John, "Biomedical Image Transmission Based on Modified Feistal Algorithm," International Journal of Computer Science \& Information Technology, vol/issue: 5(3), pp. 175-182, 2013.

[11] V. Tiwari, et al., "Performance Evaluation of Various Compression Techniques on Medical Images," International Journal of Advanced Electronics and Communication Systems, vol/issue: 1(2), 2012.

[12] K. Gupta, et al., "Lossless Medical Image Compression using Predictive Coding and Integer Wavelet Transform based on Minimum Entropy Criteria," International Journal of Application or Innovation in Engineering \& Management, vol/issue: 2(8), pp. 98-106, 2013.

[13] V. Kunchigi, et al., "Simulation of Vedic Multiplier in DCT Applications," International Journal of Computer Applications, vol/issue: 63(16), pp. 27-32, 2013.

[14] J. Sarala and E. Sivanantham, "Design of Multilevel Two Dimensional-Discrete Wavelet Transform For Image Processing Applications," International Journal of Computing Communication and Information System, vol/issue: 6(1), pp. 1-6, 2014.

[15] T. Sowjanya and N. S. Babu, "A High Performance Video Transform Engine By Using Space-Time Scheduling Strategy Reversible Vedic Gates," International Journal of Electronics and Electrical. Eng \& Telecoms, vol/issue: 3(4), pp. 95-100, 2014.

[16] I. Scholl, et al., "Challenges of medical image processing," Springer, pp. 5-13, 2011.

[17] N. Wilt, "The CUDA Handbook: A Comprehensive Guide to GPU Programming," Addison-Wesley, Computers, 2013.

[18] M. Summerfield, “Advanced QT Programming: Creating Great Software with C++ and QT 4,” Addison-Wesley, Computers, 2011.

[19] M. Scarpino, "OpenCL in Action: How to Accelerate Graphics and Computation," Manning, Computers, 2012.

[20] Suma and V. Sridhar, "Article: A Review of the Effective Techniques of Compression in Medical Image Processing," International Journal of Computer Applications, vol/issue: 97(6), pp. 23-30, 2014.

[21] "Public Image Databases," Cornell University Vision and Image Analysis Group, Retrieved, 29 july, 2015. Link:http://www.via.cornell.edu/databases/

\section{BIOGRAPHIES OF AUTHORS}

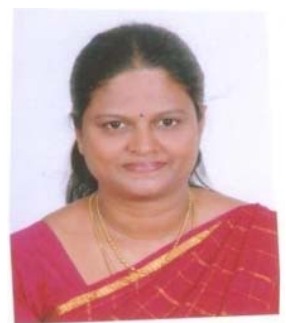

Suma has obtained her B.E. in E\&C engg from AIT, Chikmagalur-Kuvempu University, Karnataka, M.Tech from SJCE, Mysore, pursuing Ph.D at PET Research centre, PESCE, Mandya, Karnataka. She is serving as Associate professor in the department of E\&C, VVIET Mysore, Karnataka since 2002 and her field of academic interests are Digital Image processing, VLSI Design, Digital Circuits, HDL, Low power design,. She has published 2 Research Papers in International Journals. Several Projects have been guided for UG \& PG Students.

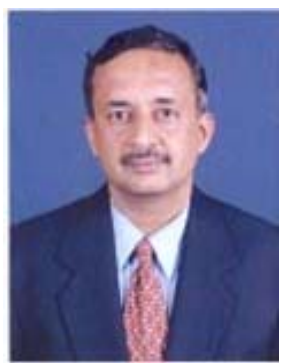

Dr. V. Sridhar has obtained his B.E. in E\&C engg from PESCE Mandya-University of Mysore, M.E. from Calcutta- Jadavpur University, Ph.D from Centre for Biomedical Engineering. Indian Institute of Technology, New Delhi ,awarded during 1995 and POST DOCTORAL RESEARCH from University of Tenaga National (UNITEN), Kajang, MALAYSIA from 2000 to 2002.He is serving this institution as Principal since 1st May 2008 and his field of academic interests are VLSI Design, Digital signal processing, Bio medical Engineering \& instrumentation and cognitive studies, Computer and Mobile communications. He has published 12 Research Papers, 6 International Journals \& 1 national journal. Several Projects have been guided for UG \& PG Students. Has organized several short courses and workshops. 